\title{
EDITORIAL
}

\section{Extensively drug-resistant tuberculosis: back to the future}

\author{
G.B. Migliori*, G. Sotgiü ${ }^{\#}$ C. Lange ${ }^{\star}$ and R. Centis*
}

$\mathbf{T}$ uberculosis (TB) has been found in mammoth bones and in Egyptian mummies. It has affected mankind since its appearance, despite many efforts to control and eliminate it.

Epidemiology indicates that, in the absence of any intervention (e.g. under the natural history model), one infectious case is likely to infect $\sim 10$ persons $\cdot \mathrm{yr}^{-1}$ for $2 \mathrm{yrs}$, thus generating 20 infected individuals [1]. Given that the lifetime breakdown is estimated to be $10 \%$, and that $\sim 50 \%$ of cases are likely to become sputum smear-positive, one infectious source is likely to generate another infectious (sputum smear-positive) case [1]. This is in the absence of intervention (fig. 1a). The interventions recommended by the directly observed treatment, short course (DOTS) strategy and the Stop TB Strategy [2] (e.g. rapid diagnosis of $70 \%$ of existing sputum smearpositive cases and effective treatment of $85 \%$ of cases) are very powerful in modifying this cycle (fig. 1b). The period of infectiousness is reduced by $\leqslant 6$ months and the number of infected individuals is reduced to only five. Given the same breakdown rate and the same risk of developing a sputum smear-positive form of TB, one infectious case produces only a quarter the number of infectious cases of TB. In other words, it takes four infectious cases to produce one new one.

If the TB programme is able to reduce transmission so dramatically, one can expect a levelling off of the epidemic curve in a given setting after 5-7 yrs following implementation of the Stop TB Strategy. This has been demonstrated in countries such as Cuba and Peru. Recently it has been demonstrated that this also works very well in Europe [3]. Romania achieved $70 \%$ detection of sputum smear positive cases and $85 \%$ successful treatment of detected cases, and was able to reduce both its case load and case-fatality load after an initial increase [3].

Unfortunately, the reality is that these encouraging examples do not work in the same way in all settings. Several situations (HIV, war, malnutrition, stress and other social determinants) [4] are able to increase either the diagnostic delay/infectious period or the breakdown rate (up to $50 \%$ in the case of HIV), compromising the potentially good results expected.

\footnotetext{
*WHO Collaborating Centre for TB and Lung Diseases, Fondazione S. Maugeri, Care and Research Institute, Tradate, ${ }^{\#}$ Hygiene and Preventive Medicine Institute, University of Sassari, Sassari, Italy. "Division of Clinical Infectious Diseases, Medical Clinic, Research Center Borstel, Borstel, Germany.

CORRESPONDENCE: G.B. Migliori, WHO Collaborating Centre for TB and Lung Diseases, Fondazione S. Maugeri, Care and Research Institute, via Roncaccio 16, 21049, Tradate, Italy. E-mail: giovannibattista.migliori@fsm.it
}

In sub-Saharan Africa, for example, in the absence of systematic use of antiretroviral drugs, even the best DOTS programme cannot achieve better results than those in figure 1 . The sum of all these factors explains why, although we have a good strategy and effective drugs, TB is still with us, even in rich and developed European countries. The main interventions used to control TB are summarised in figure 2, from the discovery of the TB bacillus to the description of extensively drug-resistant (XDR)-TB.

The first sanatorium was opened in Germany in 1857 before $\mathrm{R}$. Koch discovered in 1882 that Mycobacterium tuberculosis was the causative agent of $\mathrm{TB}$ [5]. It was clear at the time that simply isolating infectious cases in remote sanatoria in the mountains, thereby removing sources of infection from the community, had a positive effect on public health, in addition to the individual benefit represented by providing good food, rest, sun and fresh air as a contribution to helping the immune system fight the disease. The introduction of the dispensary system in 1897 in Scotland, UK, further improved the system's ability to detect and isolate infectious cases in the community and treat them. After 1907, when C. Forlanini demonstrated that artificial pneumothorax further increased the chances of a cure by creating a difficult environment for the bacilli (which are aerobes), sanatoria increased their surgical support. The drug period started later, at the end of the Second World War, when streptomycin proved to be effective against the bacillus. Unfortunately, alongside initial positive results, drug resistance appeared and multi-drug chemotherapy regimens were gradually introduced to limit this phenomenon and to increase the chances of a cure. Almost at the same time, the bacille Calmette-Guérin vaccination was introduced as a component of TB-control programmes at birth and then in different settings, with one or more revaccinations, even though no clear evidence on the effectiveness of revaccination was made available [6].

A brief comment is deserved for the idea, very popular in the 1950s, that early radiological screening of the general population would allow detection of initial forms of TB, thus allowing early and effective treatment in the pre-clinical stage of $\mathrm{TB}$, with subsequent eradication of the disease. Unfortunately, the reality was rather different [7]. Even by screening the general population every $2 \mathrm{yrs}$, the majority of the cases detected were in patients self-reporting to health services because of signs and symptoms of the disease. Despite regular and frequent time intervals between screenings ( 2 yrs or even 1 yr), TB was able to appear between the screenings. This strategy was 
a)

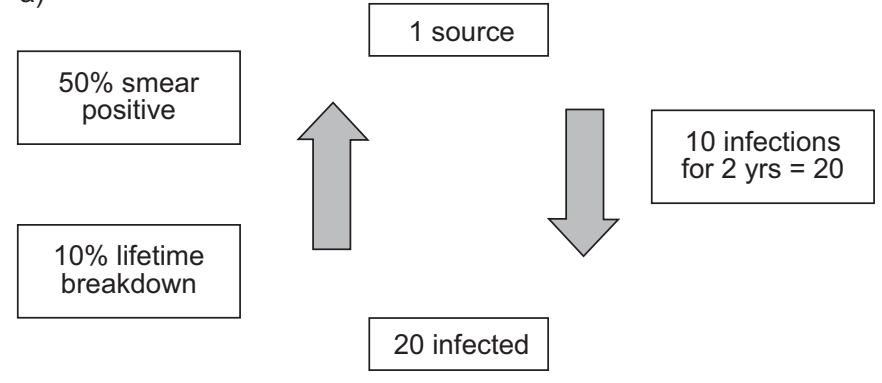

b)

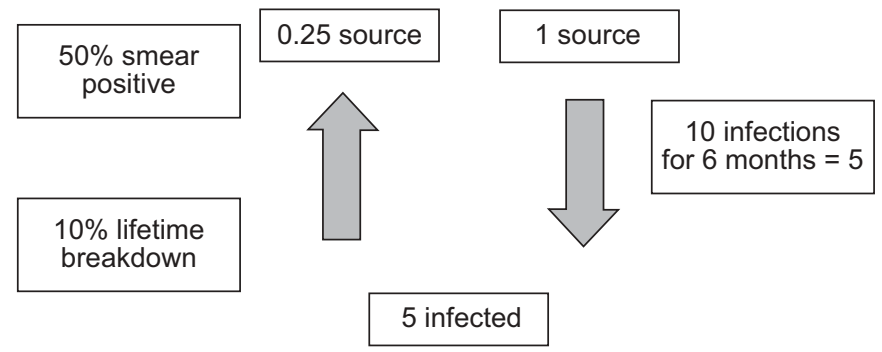

FIGURE 1. Tuberculosis transmission model in a) the absence of control interventions and b) the application of early diagnosis and effective treatment.

gradually abandoned due to its relatively low effectiveness and the high cost of running the screenings.

Recently, the modern strategy to control and eliminate TB in the low-incidence European setting was based on risk-group management and outbreak management [8]. Despite the gradual decrease in TB incidence in the general population (fig. 2), TB was still prevalent in definite risk groups thus requiring a specific intervention strategy. Furthermore, outbreaks were also becoming common, requiring contact tracing in concentric circles (the stone-in-the-pond principle) [9] and preventive chemotherapy for infected individuals to prevent future sources of infectious TB from occurring.

As can be seen in figure 2, although socioeconomic improvement still needs to continue (with poverty still being the most important determinant of the disease), as does drug therapy (hopefully supported by the introduction of new potent drugs as well as of new more effective vaccines), the era of general screening is definitively over. The question now is whether sanatoria have outlived their usefulness or not. It seems apparent that they have because the vast majority of sanatoria were closed in the 1970s and 1980s.

Unfortunately, the emergence of XDR-TB $[10,11]$ has brought us back to the pre-antibiotic era. For the first time the increased risk (relative risk 5.5) of death of XDR-TB cases compared to multidrug-resistant (MDR)-TB cases has been described [12], as well as the first two cases resistant to all known drugs [13]. For these cases the acronym XXDR-TB has been proposed [5, 13 ] in order to identify cases that are virtually untreatable with drugs. These cases are offered the limited treatment weapons available in the pre-antibiotic era; that is, good food, fresh air and, perhaps, artificial pneumothorax.

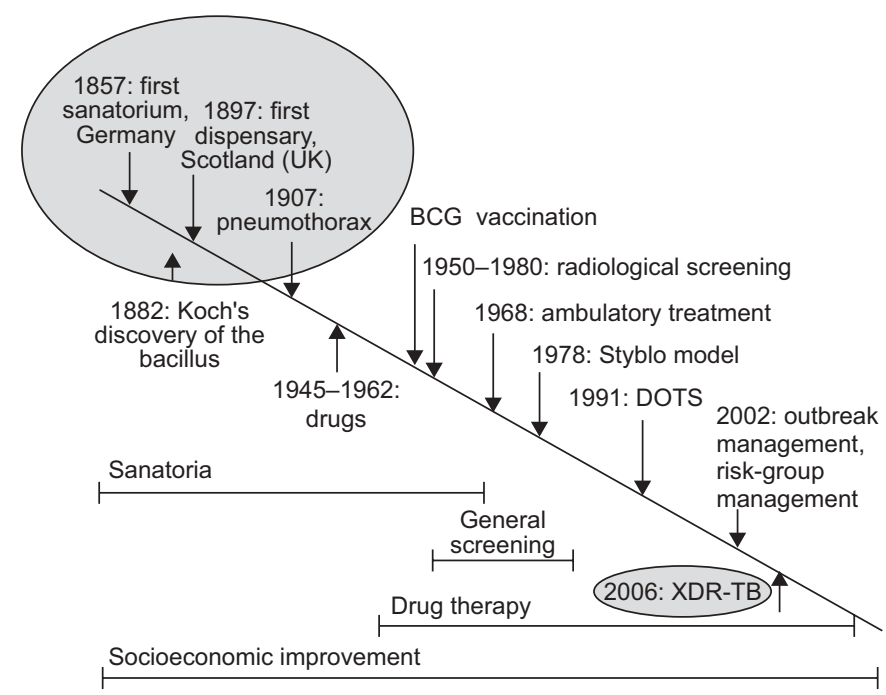

FIGURE 2. The history of tuberculosis (TB) control interventions shown over the declining curve of TB incidence in a generic country. BCG: bacille CalmetteGuérin; DOTS: directly observed treatment, short course; XDR-TB: extensively drugresistant-TB.

These patients (who are now becoming common in increasingly more countries) will die within a few (or sometimes several) months. Can we allow them to stay at home, where other relatives live and where intensive care is difficult to provide? Can we afford to admit them to hospital wards and to expose other patients, healthcare staff and visitors to the possibility of infection? Is the patient's individual freedom to decide what to do prevailing over the right of public health to protect the community from XDR-TB strains?

These are topics being discussed increasingly frequently among specialists as well as in the media, and these issues are solved in different ways in different countries. The main fact here is that the use of sanatoria may be on the horizon again, although with many differences compared to those of the past.

The interesting manuscript by LEIMANE et al. [14] sheds more light on the complicated, and still largely unknown, issue of XDR-TB. The authors revised the outcomes of 1,027 MDR-TB cases (48 of them being XDR) diagnosed nationwide in Latvia between 2002 and 2004. While the success rate of MDR-TB cases increased over time, testifying improved programme performances, the success rate of XDR-TB cases remained low $(38 \%)$. The authors also identified the characteristics associated with poor outcomes (retired status, bilateral cavitations, previous MDR-TB treatment if aged $\geqslant 55 \mathrm{yrs}$ ) and confirmed the validity of the World Health Organization recommendation to prescribe these cases a minimum of four effective drugs. The findings of this study ideally follow and complement those of the previous TBNET study validating the XDR-TB definition [10] and other studies [11] testifying the European Respiratory Journal leadership in the field.

\section{STATEMENT OF INTEREST}

None declared. 


\section{REFERENCES}

1 Styblo K, Meijer J, Sutherland I. Tuberculosis Surveillance Research Unit Report No. 1: the transmission of tubercle bacilli; its trend in a human population. Bull Int Union Tuberc 1969; 42: 5-104.

2 Raviglione MC, Uplekar M. WHO's new Stop TB Strategy. Lancet 2006; 367: 952-955.

3 Marica C, Didilescu C, Galie N, et al. Reversing the tuberculosis upwards trend: the Romanian success story. Eur Resp J 2009; 33: 168-170.

4 Lönnroth K, Jaramillo E, Williams BG, et al. Drivers of tuberculosis epidemics: the role of risk factors and social determinants. Soc Sci Med 2009; 68: 2240-2246.

5 Migliori GB, Loddenkemper R, Blasi F, et al. 125 years after Robert Koch's discovery of the tubercle bacillus: the new XDR-TB threat. Is "science" enough to tackle the epidemic? Eur Resp J 2007; 29: 423-427.

6 Rieder HL, ed. Intervention for tuberculosis control and elimination. 2002. International Union Against Tuberculosis and Lung Disease, Paris. www.tbrieder.org/publications/interventions_en.pdf

7 Krivinka R, Drapela J, Kubik A, et al. Epidemiological and clinical study of tuberculosis in the district of Kolin, Czechoslovakia. Bull World Health Org 1974; 51: 59-69.
8 Broekmans JF, Migliori GB, Rieder HL, et al. European framework for tuberculosis control and elimination in countries with a low incidence. Recommendations of the World Health Organization (WHO), International Union against Tuberculosis and Lung Disease (IUATLD) and Royal Netherlands Tuberculosis Association (KNCV) Working Group. Eur Respir J 2002; 19: 765-775.

9 Erkens CGM, Kamphorst M, Abubakar I, et al. Tuberculosis contact investigation in low prevalence countries: a European consensus. Eur Respir J 2010; [In press DOI: 10.1183/ 09031936.00201609].

10 Migliori GB, Besozzi G, Girardi E, et al. Clinical and operational value of the extensively drug-resistant tuberculosis definition. Eur Resp J 2007; 30: 623-626.

11 Sotgiu G, Ferrara G, Matteelli A, et al. Epidemiology and clinical management of XDR-TB: a systematic review by TBNET. Eur Respir J 2009; 33: 871-881.

12 Migliori GB, Ortmann J, Girardi E, et al. Extensively drug-resistant tuberculosis, Italy and Germany. Emerg Infect Dis 2007; 13: 780-782.

13 Migliori GB, De Iaco G, Besozzi G, et al. First tuberculosis cases in Italy resistant to all tested drugs. Euro Surveill 2007; 12: E070517.1.

14 Leimane V, Dravniece G, Riekstina V. Treatment outcome of multidrug/extensively drug-resistant tuberculosis in Latvia, 20002004. Eur Respir J 2010; 36; 584-593. 